\title{
Impact of sensory processing dysfunction on fine motor skills in autism spectrum disorders
}

\author{
DOI: https://doi.org/10.5114/pq.2020.100277
}

\author{
Rajeswari Muthusamy', Ramachandran Padmanabhan', Binu Ninan', Sailakshmi Ganesan² \\ ${ }^{1}$ Sri Ramachandra Institute of Higher Education and Research, Chennai, India \\ 2 Spastics Society of Tamilnadu, Chennai, India
}

Abstract

Introduction. Children with autism spectrum disorders predominantly exhibit social impairment but significant physical features are manifested as motor delays and deficits affecting their daily living. Though sensory integration is a basic component required for motor skills, the impact of sensory processing dysfunction on fine motor skills is not clear, which is explored in this study.

Methods. This cross sectional study was carried out in Vidya Sudha - school for children with special needs. Overall, 56 children diagnosed with autism spectrum disorders with the Childhood Autism Rating Scale were included in the study. Peabody Developmental Motor Scale-2 was used to assess the level of their fine motor skill, and the sensory profile served to evaluate their sensory processing dysfunction.

Results. Pearson's correlation showed a strong positive correlation between the fine motor quotient and auditory, visual, vestibular, and tactile processing, with $r>0.7$ and $p \leq 0.05$. Beta value of logistic regression of tactile, vestibular, and visual processing was $0.554,0.288$, and 0.191 , respectively, which conveys that tactile dysfunction has a major impact, followed by vestibular and visual processing deficits.

Conclusions. The study concludes that tactile, vestibular, and visual sensory dysfunctions appear to influence the fine motor skills, with tactile dysfunction exerting a greater impact. The result strongly emphasizes that paediatric therapists should individualize treatment on the basis of sensory dysfunction, which should be considered and simultaneously addressed when training fine motor function and activities of daily living.

Key words: autism spectrum disorder, sensory profile, fine motor skill, Peabody Developmental Motor Scale, sensory processing dysfunction

\section{Introduction}

Autism spectrum disorder (ASD) is a heterogeneous condition that affects social communication and social interactions, with restricted, repetitive, stereotyped patterns of behaviour, interests, and activities. The symptoms together limit and impair everyday functioning. ASD is a diverse condition affecting groups of individuals with a wide variety of disability and intellectual dysfunction [1]. ASD is a neurodevelopmental disorder with a serious developmental disadvantage to the child in the form of poor schooling and social function; it also affects adult productivity [2]. Globally, the incidence of autism is found to be $62 / 10,000$ and there was an increasing incidence from 1998 to 2015, which reflects an increased level of recognition and documentation [3, 4]. According to WHO, 1 in 160 children had ASD in the year 2019, and an estimated prevalence of ASD in a selected population of school children in India was established to be $0.23 \%$, whereas the prevalence in European countries ranged from $0.33 \%$ to $3.13 \%[5,6]$. Careful diagnosis and interdisciplinary approach with cooperation are mandatory to shorten the suffering of the ASD population [7].

Although ASD is considered a psychiatric disorder, physical features are associated with it and motor impairments in these individuals have been categorized as 'associated symptoms'. Hypotonia, motor apraxia, reduced ankle mobility, history of gross and fine motor delay and toe-walking are the motor deficits clinically found in children with ASD [8]. These children showed similar deficits in gross and fine motor skills (FMS) as children with developmental delay, which indicates that all children with ASD should receive complete developmental evaluation, including assessment of their motor functioning [9]. Gross and fine motor assessment and programmes should be part of the early intervention plans to prevent further decline and isolation from social interaction with peers [10]. An empirical support for a direct link between motor and social impairments in ASD was found, which brought about general and specific implications for physical therapy [11]. The mean age of parental recognition of any abnormality in a child with ASD was 23.4 months, with a mean time lag of 4 months to seek professional help, and the final diagnosis established at 32 months of age [12]. Variations from typical development and delayed language progress are identified in many children with ASD by 24 months of age, and unusual slowing in performance occurred between 14 and 24 months of age [13].

Literature reveals that ASD children present gross and fine motor delay; gross motor deficits improve over time and the child gains motor independence at the time they reach for professional help but deficits in FMS interfere with the children's activities of daily living even after interventional therapy, thereby blocking the child's development. Reaching and grasping appear to be delayed in infants at risk for ASD. A mean delay of 8 months in FMS was observed over the first

Correspondence address: Rajeswari Muthusamy, Sri Ramachandra Institute of Higher Education and Research, Ramachandra Nagar, Porur Chennai 600116, India, e-mail: rajeswari@sriramachandra.edu.in 
and second years of life in a range of behaviours, including clapping, pointing, playing with blocks and puzzles, and turning doorknobs [14, 15]. Motor performance in ASD children was slower and weaker in hand grip strength, which would affect their FMS [16].

Smooth, targeted, and accurate movements, both gross and fine, require a harmonious functioning of sensory input, central processing of information in the brain, and coordination with the high executive cerebral functions [17]. Sensory integration is the ability to receive information through the senses of touch, movement, smell, taste, vision, and hearing and to combine the resulting perception with prior information, memories, and knowledge which is already stored in the brain, in order to derive coherent meaning from the processed stimuli. A distinctive pattern of sensory processing and evidence of a variety of sensory processing dysfunction (SPD) are common with ASD [18, 19]. Ninety percent of children with ASD showed some degree of SPD of under-responsiveness or sensation seeking in auditory filtering and tactile sensitivity [20]. Early onset of extreme sensory modulation behaviours in toddlers with ASD was found and 95\% of the sample demonstrated some degree of SPD and sensitivity to tactile input [21, 22].

Abnormalities in responses to sensory stimuli include both hypo- and hypersensitivity to stimuli that could be in low threshold (LT) or high threshold (HT), which inhibits participation in productive activities and burdens the activities of everyday life [23]. Though sensory integration is a basic component required for motor skills, the impact of sensory integration deficits on FMS is not clear. Hence, this study tries to explore the influence of SPD of various senses on FMS; the results would be helpful in setting realistic goals in comprehensive rehabilitation of children with ASD.

\section{Subjects and methods}

\section{Subject recruitment}

The subjects were recruited from the Vidya Sudha - school for children with special needs, Sri Ramachandra Institute of Higher Education and Research. A total of 56 children of both genders (48 males and 8 females), aged $3-5$ years (mean age: 43.03 months), diagnosed with ASD with the Childhood Autism Rating Scale were included in the study. Subjects who were associated with attention deficit hyperactivity disorder signs, upper limb musculoskeletal injuries, or with visual or auditory impairments were excluded from the study.

\section{Instrumentation}

\section{Peabody Developmental Motor Scale-2}

The Peabody Developmental Motor Scale, $2^{\text {nd }}$ edition (PDMS-2) is composed of 6 subtests that measure interrelated abilities in early motor development. It was designed to assess gross motor skills and FMS in children from birth through 6 years of age [24]. The subtest measures are: reflexes, stationary, locomotion, object manipulation, grasping, and visual motor integration. All of the PDMS-2 subtests contribute to the total motor quotient, which is considered as the best estimate of overall motor abilities. The grasping and visual motor integration subtests contribute to the fine motor quotient (FMQ) score.

The grasping subtest measures a child's ability to use their hands. It begins with the ability to hold an object with one hand and progresses up to actions involving the controlled use of the fingers of both hands to button and unbutton garments.

The visual motor integration subtest measures a child's ability to use their visual perceptual skills to perform complex eye-hand coordination tasks, such as reaching for and grasping an object, building with blocks, and copying designs.

\section{Sensory profile}

The sensory profile provides a standard method to measure a child's sensory processing abilities and to profile the effect of sensory processing on functional performance in the daily life of a child. The sensory profile is a judgment-based caregiver questionnaire. Each item describes the child's response to a particular sensory experience [25]. The caregiver who has daily contact with the child completes the questionnaire by reporting the frequency with which these behaviours occur (always, frequently, occasionally, seldom, or never). The professional then scores the responses in the questionnaire.

\section{Procedure}

Children diagnosed with ASD by a psychologists using the Childhood Autism Rating Scale were included in the study after due informed consent from their parents or caretakers. PDMS-2 was used to assess the level of their fine motor development; depending on the chronological age, the items were administered in each subtest in accordance with the guidelines provided in the manual, and the raw score was calculated. The obtained raw score for each subtest was converted to age equivalent, percentile, and standard. The sum of the standard scores for the fine motor subscale was converted to $F M Q$. On the basis of the standard scores and quotient scores, the infant motor development was ranked as very superior, superior, above average, average, below average, poor, or very poor. After the assessment with the sensory profile, where the caregiver answered the questionnaire to evaluate the child's sensory processing deficits, the components were scored accordingly.

\section{Data analysis}

The data were analysed with the SPSS software, version 17.0. Pearson's correlation was used to find the relationship of FMS with LT and HT of various senses, and backward logistic regression served to determine the SPD which predominantly influenced FMS in ASD.

\section{Ethical approval}

The research related to human use has complied with all the relevant national regulations and institutional policies, has followed the tenets of the Declaration of Helsinki, and has been approved by the Ethics Committee of Sri Ramachandra Institute of Higher Education and Research (IEC/ $\mathrm{NI} / 14 / \mathrm{JAN} / 38 / 11$ ).

\section{Informed consent}

Informed consent has been obtained from the legal guardians of all individuals included in this study.

\section{Results}

Table 1 presents the demographic characteristics of the investigated children and the sensory processing results. 
Table 1. Study participants' demographics and sensory processing results

\begin{tabular}{|c|c|}
\hline Factors & Mean $(S D)$ \\
\hline Age & 43.03 (3.35) months \\
\hline Gender & $\begin{array}{l}\text { Males: } n=48 \\
\text { Females: } n=8\end{array}$ \\
\hline Fine motor quotient & $69.86(9.422)$ \\
\hline Auditory processing & $22.41(5.546)$ \\
\hline Visual processing & 29.39 (5.119) \\
\hline Vestibular processing & $38.59(6.161)$ \\
\hline Tactile processing & $52.48(7.006)$ \\
\hline Multisensory processing & $22.55(4.268)$ \\
\hline Oral processing & $42.13(7.025)$ \\
\hline
\end{tabular}

LT and HT subscales scores of auditory, visual, vestibular, and tactile dysfunction were positively correlated with FMS, with a statistically significant $p$ value of $<0.05$ (Table 2 ).

Tactile, visual, and vestibular dysfunctions had a highly significant impact on FMS. Auditory dysfunction was found to exert an influence but comparatively lesser than the previous 3 factors. Multisensory and oral processing did not have an impact on FMS (Table 3).

\section{Discussion}

Children with ASD become anxious when they respond to and interpret their environment. As the child attempts to process the sensory information, their ability to concentrate and stay focused may become impaired. Fine motor activity demands focusing and attention, but attention becomes distracted in ASD children owing to various sensory processing abnormalities which make the processing slow, inconsistent, or abnormal. The purpose of this study was to

Table 2. Correlations of fine motor quotient and sensory processing

\begin{tabular}{|c|c|c|c|c|c|c|c|}
\hline Factors & & & $n$ & Mean & $S D$ & $r$ (correlation with $\mathrm{FMQ}$ ) & $p$ \\
\hline FMQ & & & & 69.86 & 9.422 & & \\
\hline & & LT & & 17.67 & 4.70 & 0.701 & 0.00 \\
\hline & & HT & & 4.73 & 1.78 & 0.617 & 0.00 \\
\hline & & LT & & 23.58 & 4.45 & 0.684 & 0.00 \\
\hline & visuar processilig & HT & & 5.80 & 1.60 & 0.527 & 0.00 \\
\hline & Yontibular nromenging & LT & & 23.39 & 3.43 & 0.620 & 0.00 \\
\hline & vectiverar prosucomig & HT & 56 & 15.16 & 4.00 & 0.660 & 0.00 \\
\hline 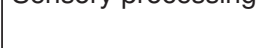 & Tontile nromenging & LT & & 29.41 & 5.62 & 0.771 & 0.00 \\
\hline & 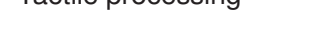 & HT & & 23.07 & 3.32 & 0.526 & 0.00 \\
\hline & Multiconsory nrococsing & $\mathrm{LT}$ & & 2.91 & 0.96 & 0.334 & 0.01 \\
\hline & 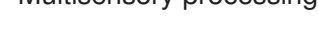 & HT & & 13.87 & 3.11 & 0.378 & 0.04 \\
\hline & & LT & & 15.85 & 3.61 & 0.244 & 0.06 \\
\hline & & $\mathrm{HT}$ & & 26.26 & 5.42 & 0.018 & 0.89 \\
\hline
\end{tabular}

FMQ - fine motor quotient, LT - low threshold, HT - high threshold

Table 3. Logistic regression coefficients

\begin{tabular}{|c|c|c|c|c|c|c|c|}
\hline \multirow{2}{*}{ Model } & \multicolumn{2}{|c|}{ Unstandardized coefficients } & \multirow{2}{*}{$\begin{array}{c}\text { Standardized } \\
\text { coefficients }\end{array}$} & \multirow{2}{*}{$\mathrm{T}$} & \multirow{2}{*}{ Significance } & \multicolumn{2}{|c|}{$95.0 \%$ confidence interval for B } \\
\hline & B & Standard error & & & & Lower bound & Upper bound \\
\hline (Constant) & 8.397 & 4.796 & & 1.751 & 0.086 & -1.241 & 18.035 \\
\hline Auditory processing & 0.177 & 0.167 & 0.104 & 1.060 & 0.294 & -0.158 & 0.511 \\
\hline Visual processing & 0.348 & 0.144 & 0.189 & 2.424 & 0.019 & 0.059 & 0.636 \\
\hline Vestibular processing & 0.473 & 0.135 & 0.310 & 3.510 & 0.001 & 0.202 & 0.744 \\
\hline Tactile processing & 0.666 & 0.141 & 0.495 & 4.720 & 0.000 & 0.382 & 0.949 \\
\hline Multisensory processing & -0.141 & 0.152 & -0.064 & -0.926 & 0.359 & -0.446 & 0.165 \\
\hline Oral processing & -0.065 & 0.071 & -0.049 & -0.916 & 0.364 & -0.209 & 0.078 \\
\hline (Constant) & 3.442 & 3.749 & & 0.918 & 0.363 & -4.081 & 10.965 \\
\hline Visual processing & 0.351 & 0.145 & 0.191 & 2.428 & $0.019^{*}$ & 0.061 & 0.641 \\
\hline Vestibular processing & 0.441 & 0.120 & 0.288 & 3.661 & $0.001^{*}$ & 0.199 & 0.682 \\
\hline Tactile processing & 0.745 & 0.101 & 0.554 & 7.342 & $0.000^{*}$ & 0.541 & 0.948 \\
\hline
\end{tabular}


determine the impact of SPD on FMS in children with ASD. The mean FMQ was found to be 69.86 , which shows that the subjects had poor performance in grasping and visual motor integration. This result was similar to the observation by Provost et al. [26], who compared the level of gross motor and fine motor development in children with ASD and concluded that the motor profile of children with ASD was analogous to that of children with developmental delay who did not have ASD.

The mean score for auditory, vestibular, tactile, and multisensory processing was $22.41,38.59,52.48$, and 22.55 , respectively, which indicates a definite difference in these components; the mean score for visual and oral processing was 29.39 and 42.13, respectively, which implies a probable difference in sensory processing leading to atypical performance with reference to normal children. This finding is in line with the results of a cross-sectional study performed by Kern et al. [27], who suggested that autistic people presented abnormal auditory, visual, tactile, and oral sensory processing as compared with the control group and that the deficits were global in nature and had a potential to improve with age.

The correlation between FMQ and SPD in LT and HT stimuli was positive for all senses except oral processing, which is supported by a study by Liu [28], who revealed that delayed sensory processing of visual, auditory, and tactile stimuli was related to gross and fine motor difficulties in children with ASD. Tactile defensiveness may prevent manipulating objects and the decreased body awareness due to tactile processing dysfunction impairs learning to grasp objects efficiently. This is evident in the strong correlation of tactile processing of LT stimuli and $F M Q$, with $r$ value of 0.77 . The result is supported by a study performed by Case-Smith [29], in which children with both defensiveness and discrimination problems demonstrated the least efficiency in all hand manipulation tasks and had significantly higher time scores on the turn and translation in hand manipulation tasks.

Children with vestibular processing dysfunction face difficulty in stabilizing their trunk, shoulder, and arms while using an upper limb for grasping objects and respond negatively to unexpected or loud distracting noises. FMS require concentration and focus on task, and this processing dysfunction may distract the child from completing the task; thereby, activities of daily living are also impaired. White et al. [30] measured the occupational performance in children with SPD and made comparisons with the sensory profile. They found that the subjects faced challenges in performing everyday occupations, which indicates that SPD exerts a direct effect on fine motor activity.

The regression analysis showed that tactile dysfunction, followed by vestibular and visual processing dysfunction, most influenced FMS in ASD. Tactile perception refers to the passive contact of the skin with an object, and haptic perception occurs when the child actively explores and manipulates objects with the hands. Children with ASD might also have reduced haptic perception along with LT and HT for stimulus, which contributes to the overall motor performance. A study by Riquelme et al. [31] showed by using somatosensory assessments that discriminative touch and pressure pain of upper limbs in children with ASD were significantly altered compared with those in typically developing children, which could also indicate an increased impact of tactile processing on FMS. As found by Glazebrook et al. [32], individuals with autism used vision and proprioception in executing manual reaching movements and they took considerably more time to perform movements that required greater visual-proprioceptive integration; this could be due to the effect of visual processing dysfunctions on FMS, as evident in this study. SPD affects the motor performance of an ASD child, which might influence the child's ability to participate in activities of daily living. This finding must be taken into account when framing goals during therapeutic intervention by individualizing the treatment on the basis of SPD and simultaneously addressing SPD when training FMS.

\section{Limitations}

Some tasks in the fine motor component, e.g. using scissors, had not been previously explored by the children, which affected the score in PDMS-2. The motor skill scores of PDMS-2 were based on observation of performance, which was difficult to assess as children with ASD have decreased imitation skills; the assessment was therefore time-consuming.

\section{Conclusions}

The results of this study imply that SPD in the tactile, visual, and vestibular processing has the greatest impact on FMS, which conveys that increased SPD reduces FMS. The study strongly emphasizes that paediatric therapist should individualize treatment on the basis of SPD, which should be considered and simultaneously addressed when training fine motor function in children with ASD. They gradually learn to organize and give meaning to sensory inputs, which helps to focus on appropriate sensation, changing the quality of movement from jerky or clumsy to more refined and thereby improving the activities of daily living.

\section{Disclosure statement}

No author has any financial interest or received any financial benefit from this research.

\section{Conflict of interest}

The authors state no conflict of interest.

\section{References}

1. Diagnostic and statistical manual of mental disorders, $5^{\text {th }}$ ed. American Psychiatric Association; 2013.

2. Dalwai S, Ahmed S, Udani V, Mundkur N, Kamath SS, Nair MKC. Consensus statement of the Indian Academy of Pediatrics on evaluation and management of autism spectrum disorder. Indian Pediatr. 2017;54(5):385393; doi: 10.1007/s13312-017-1112-4.

3. Elsabbagh M, Divan G, Koh Y-J, Kim YS, Kauchali S, Marcín C, et al. Global prevalence of autism and other pervasive developmental disorders. Autism Res. 2012; 5(3):160-179; doi: 10.1002/aur.239.

4. Myers SM, Voigt RG, Colligan RC, Weaver AL, Storlie CB, Stoeckel RE, et al. Autism spectrum disorder: incidence and time trends over two decades in a population-based birth cohort. J Autism Dev Disord. 2019;49(4):1455-1474; doi: 10.1007/s10803-018-3834-0.

5. Rudra A, Belmonte MK, Soni PK, Banerjee S, Mukerji S, Chakrabarti B. Prevalence of autism spectrum disorder and autistic symptoms in a school-based cohort of children in Kolkata, India. Autism Res. 2017;10(10):15971605; doi: 10.1002/aur.1812.

6. Delobel-Ayoub M, Saemundsen E, Gissler M, Ego A, Moilanen I, Ebeling $\mathrm{H}$, et al. Prevalence of autism spectrum disorder in 7-9-year-old children in Denmark, Finland, France and Iceland: a population-based registries approach within the ASDEU project. J Autism Dev Disord. 2020;50(3):949-959; doi: 10.1007/s10803-019-04328-y. 
7. Brzezicka DA, Kucia K. Deficit of empathy: antisocial personality or autism spectrum disorder - differential diagnosis [in Polish]. Neuropsychiatr Neuropsychol. 2018;13(4): 135-141; doi: 10.5114/nan.2018.83037.

8. Ming X, Brimacombe M, Wagner GC. Prevalence of motor impairment in autism spectrum disorders. Brain Dev. 2007;29(9):565-570; doi: 10.1016/j.braindev.2007.03. 002.

9. Provost B, Lopez BR, Heimerl S. A comparison of motor delays in young children: autism spectrum disorder, developmental delay, and developmental concerns. J Autism Dev Disord. 2007;37(2):321-328; doi: 10.1007/ s10803-006-0170-6.

10. Lloyd M, MacDonald M, Lord C. Motor skills of toddlers with autism spectrum disorders. Autism. 2013;17(2): 133-146; doi: 10.1177/1362361311402230.

11. Bhat AN, Landa RJ, Galloway JC. Current perspectives on motor functioning in infants, children, and adults with autism spectrum disorders. Phys Ther. 2011;91(7):1116 -1129; doi: 10.2522/ptj.20100294.

12. Chakrabarti S. Early identification of autism. Indian Pediatr. 2009;46(5):412-414.

13. Landa R, Garrett-Mayer E. Development in infants with autism spectrum disorders: a prospective study. J Child Psychol Psychiatry. 2006;47(6):629-638; doi: 10.1111/ j.1469-7610.2006.01531.x.

14. Gernsbacher MA, Sauer EA, Geye HM, Schweigert EK, Hill Goldsmith H. Infant and toddler oral- and manualmotor skills predict later speech fluency in autism. J Child Psychol Psychiatry. 2008;49(1):43-50; doi: 10.1111/j. 1469-7610.2007.01820.x.

15. Lane A, Harpster K, Heathcock J. Motor characteristics of young children referred for possible autism spectrum disorder. Pediatr Phys Ther. 2012;24(1):21-29; doi: 10.1097/pep.0b013e31823e071a.

16. Hardan AY, Kilpatrick M, Keshavan MS, Minshew NJ. Motor performance and anatomic magnetic resonance imaging (MRI) of the basal ganglia in autism. J Child Neurol. 2003;18(5):317-324; doi: 10.1177/0883073803 0180050801.

17. Sharkey J. The concise book of neuromuscular therapy. A trigger point manual. Chichester: Lotus Publishing; 2008.

18. Watling RL, Deitz J, White O. Comparison of sensory profile scores of young children with and without autism spectrum disorders. Am J Occup Ther. 2001;55(4):416423; doi: 10.5014/ajot.55.4.416.

19. Schaaf RC, Benevides T, Mailloux Z, Faller P, Hunt J, van Hooydonk $E$, et al. An intervention for sensory difficulties in children with autism: a randomized trial. J Autism Dev Disord. 2014;44(7):1493-1506; doi: 10.1007/s10803013-1983-8.

20. Schauder KB, Bennetto L. Toward an interdisciplinary understanding of sensory dysfunction in autism spectrum disorder: an integration of the neural and symptom literatures. Front Neurosci. 2016;10:268; doi: 10.3389/ fnins.2016.00268.

21. Ben-Sasson A, Cermak SA, Orsmond GI, Tager-Flusberg $\mathrm{H}$, Carter AS, Kadlec MB, et al. Extreme sensory modulation behaviors in toddlers with autism spectrum disorders. Am J Occup Ther. 2007;61(5):584-592; doi: 10.5014/ajot.61.5.584.

22. Tomchek SD, Dunn W. Sensory processing in children with and without autism: a comparative study using the short sensory profile. Am J Occup Ther. 2007;61(2):190200; doi: 10.5014/ajot.61.2.190. spectrum disorders and impact on functioning. Pediatr Clin North Am. 2012;59(1):203-214; doi: 10.1016/j. pcl.2011.10.012.

24. Folio MR, Fewell RR. Peabody Developmental Motor Scales, $2^{\text {nd }}$ ed. Austin: Pro-Ed; 2000.

25. Dunn W. Performance of typical children on the sensory profile: an item analysis. Am J Occup Ther. 1994;48(11): 967-974; doi: 10.5014/ajot.48.11.967.

26. Provost B, Heimerl S, Lopez BR. Levels of gross and fine motor development in young children with autism spectrum disorder. Phys Occup Ther Pediatr. 2007;27(3): 21-36; doi: 10.1080/j006v27n03_03.

27. Kern JK, Trivedi MH, Garver CR, Grannemann BD, Andrews AA, Savla JS, et al. The pattern of sensory processing abnormalities in autism. Autism. 2006;10(5): 480-494; doi: 10.1177/1362361306066564.

28. Liu T. Sensory processing and motor skill performance in elementary school children with autism spectrum disorder. Percept Mot Skills. 2013;116(1):197-209; doi: 10.2466/10.25.pms.116.1.197-209.

29. Case-Smith J. The effects of tactile defensiveness and tactile discrimination on in-hand manipulation. Am J Occup Ther. 1991;45(9):811-818; doi: 10.5014/ajot.45. 9.811.

30. White BP, Mulligan S, Merrill K, Wright J. An examination of the relationships between motor and process skills and scores on the sensory profile. Am J Occup Ther. 2007;61(2):154-160; doi: 10.5014/ajot.61.2.154.

31. Riquelme I, Hatem SM, Montoya P. Abnormal pressure pain, touch sensitivity, proprioception, and manual dexterity in children with autism spectrum disorders. Neural Plast.2016;2016:1723401;doi:10.1155/2016/1723401.

32. Glazebrook C, Gonzalez D, Hansen S, Elliott D. The role of vision for online control of manual aiming movements in persons with autism spectrum disorders. Autism. 2009;13(4):411-433; doi:10.1177/1362361309105659. 